\title{
Oncostatin M-induced and constitutive activation of the JAK2/STAT5/CIS pathway suppresses CCLI, but not CCL7 and CCL8 chemokine expression
} C Hintzen*1, C Haan², J Tuckermann ${ }^{3}$, PC Heinrich ${ }^{4}$ and HM Hermanns ${ }^{1}$

Address: ${ }^{1}$ Rudolf Virchow Center, DFG Research Center for Experimental Biomedicine, University of Würzburg, Würzburg, Germany, ${ }^{2}$ Life Science Research Unit, University of Luxembourg, 1511 Luxembourg, Luxembourg, ${ }^{3}$ Leibniz Institute for AgeResearch, Fritz Lipmann Institute, Jena, Germany and ${ }^{4}$ Department of Biochemistry and Molecular Biology, University of Freiburg, Freiburg, Germany

* Corresponding author

from 12th Joint Meeting of the Signal Transduction Society (STS). Signal Transduction: Receptors, Mediators and Genes Weimar, Germany. 29-31 October 2008

Published: 26 February 2009

Cell Communication and Signaling 2009, 7(Suppl I):A17 doi:I0.1I86/I478-8IIX-7-SI-AI7

This abstract is available from: http://www.biosignaling.com/content/7/SI/AI7

(c) 2009 Hintzen et al; licensee BioMed Central Ltd.

The recruitment of leukocytes to injured tissue is crucial for the initiation of inflammatory responses as well as for immune surveillance to fight tumor progression. Here we show that oncostatin $M$, a member of the interleukin-6type cytokine family and potent pro-inflammatory cytokine stimulates the expression of the chemokines CCL1, CCL7 and CCL8 in primary human dermal fibroblasts at a faster kinetic than IL-1 or TNF. The production of CCL1 and CCL8 is important for migration of monocytes, while specific antibodies against CCL1 additionally inhibit the migration of $\mathrm{T}$ lymphocytes. We identify the mitogen-activated protein kinases ERK1/2 and p38 as crucial factors for the enhanced expression of CCL1 and CCL8. Depletion of the ERK1/2 target genes c-Jun or c-Fos strongly decreases CCL1 and CCL8 expression, while p38 MAPK prolongs the half-life of CCL1, CCL7 and CCL8 mRNA through inhibition of tristetraprolin. None of the STAT transcription factors STAT1, STAT3 or STAT5 stimulate transcription of CCL1 or CCL8. However, we identify a negative regulatory function of activated STAT5 for the gene expression of CCL1. Importantly, not STAT5 itself, but its target gene CIS is required for the STAT5 inhibitory effect on CCL1 expression. Finally, we show that constitutive activation of STAT5 through a mutated form of JAK2 (JAK2 V617F) occurring in patients with myeloproliferative disorders similarly suppresses CCL1 expression. Taken together, we identify novel important inflammatory target genes of OSM, which are independent of STAT signaling per se, but depend on MAPK activation and are partly repressed through STAT5-dependent expression of CIS. 